Rich Environments, Dull Experiences: How Environment Can Exacerbate the Effect of Constraint on the Experience of Boredom

Andriy A. Struk ${ }^{\mathrm{a}}$, Abigail A. Scholer ${ }^{\mathrm{a}}$, James Danckert ${ }^{\mathrm{a}}$, Paul Seli ${ }^{\mathrm{b}}$

a. Department of Psychology

University of Waterloo

200 University Avenue West

Waterloo, ON, Canada N2M 2L9

b. Department of Psychology and Neuroscience

Duke University

417 Chapel Drive

Durham, NC, USA, 27708

Corresponding author: Andriy Struk, astruk@uwatreloo.ca

Author Note: This work was supported by Natural Sciences and Engineering Research Council of Canada under Grant \# 109618). The authors declared no conflicts of interest with respect to the authorship or the publication of this article. 


\begin{abstract}
We examined the hypothesis that boredom is likely to occur when opportunity costs are high; that is, when there is a high potential value of engaging in activities other than the researcher-assigned activity. To this end, participants were either placed in a room with many possible affordances (e.g., a laptop, puzzle, etc.; affordances condition; $n=121$ ), or they were ushered into an empty room (control condition; $n=107$ ). In both conditions participants were instructed to entertain themselves with only their thoughts (hence, participants in the affordances condition were to refrain from engaging with the available options). As predicted, participants in the affordances condition reported higher levels of boredom compared with those in the control condition. Results suggest, that under some conditions, environments that afford alternative activities may be more boring than those that are void of such activities.
\end{abstract}

Keywords: boredom, constraint, engagement, affordances 


\section{Rich Environments, Dull Experiences: How Environment Can Exacerbate the Effect of Constraint on the Experience of Boredom}

Boredom is a ubiquitous human experience. Indeed, research suggests that a typical highschool student will experience some level of boredom — up to $36 \%$ of the time - in any given day (Goetz, 2014), and that 91\% of North American youth experience boredom (The National Center on Addiction and Substance Abuse, 2003). In addition, boredom is a consequential experience linked to a slew of negative cognitive, affective, and behavioral outcomes, with inattention, depression, and substance use forming just the tip of the iceberg (for reviews see Eastwood, Frischen, Fenske, \& Smilek, 2012; Farmer \& Sundberg, 1986; Westgate \& Wilson, 2018). A thorough understanding of boredom and its antecedents is essential in the development of methods by which the consequences of boredom might be minimized, and hence, identifying such antecedents is of practical, as well as theoretical, importance. In the present study, our aim was to examine the recently theorized - yet currently untested-hypothesis that boredom results when opportunity costs are high; that is, when there is a high potential value of engaging in activities other than one's current activity (Kurzban, Duckworth, Kable \& Myers, 2013; GomezRamirez \& Costa, 2017).

Boredom is a negative emotional state commonly characterized by an inability to engage with a satisfying activity, coupled with the desire to engage in something other than what one is currently doing (Eastwood et al., 2012). Boredom is universally recognized to occur when we are stuck doing something we don't want to do, or when we find ourselves in circumstances wherein we don't know what to do (for review see Eastwood et al., 2012). This latter aspect of boredom - that we may be bored without knowing what it is that we want to do (or without 
wanting to do any of the currently available options for action)—-highlights a possible role for boredom in prompting us to identify and engage in satisfying goal pursuits.

Functional accounts of boredom suggest there is more to the experience of boredom than merely failure to engage (Danckert, Mugon, Struk \& Eastwood, 2018; Elpidorou, 2018; Bench \& Lench, 2013). Broadly speaking, these accounts suggest that boredom is an emotion that serves two functions. First, boredom informs us that the current situation is unsatisfactory in some way (most commonly represented by a lack of meaning, monotony, or inappropriate levels of challenge); second, it motivates us to alter ongoing activities or pursue new, potentially moresatisfactory goals (Elpidorou, 2018, 2018). According to this perspective, a situation is unsatisfactory (and hence, boring) when it is associated with opportunity costs (Kurzban, Duckworth, Kable \& Myers, 2013); that is, the uncomfortable feeling of boredom signals that the potential value of engaging in alternative activities is high. Thus, according to the opportunitycost framework, boredom is not solely a failure to engage, but it is also a self-regulatory signal that indicates that we could be more optimally engaged if we completed some other activity ${ }^{1}$.

In the present study, we sought to test the opportunity-cost theory of boredom by determining whether exposing participants to potential affordances promotes the experience of boredom. (Here, as per the Oxford dictionary's definition, we conceptualize "affordances" in terms of "a property of an object or an aspect of the environment, especially relating to its potential utility, which can be inferred from visual or other perceptual signals" (“Affordance,"

\footnotetext{
${ }^{1}$ We would like to note that evaluative judgements akin to those outlined by Kurzban and colleagues have been historically recognized to precede the desire or intention to pursue goals of high desirability (for review see Custers, 2009). Furthermore, although the boredom signal may motivate us to alter ongoing activities or pursue some other activity, it does not necessitate that an individual is able to respond to this signal effectively. Indeed, prior work demonstrates that trait boredom is characterized by a failure to launch into goal pursuit (Mugon, Struk, \& Danckert, 2018). This work suggests that the failure to respond to the boredom signal may be a major factor that makes the boredom experience potent and apparent to an individual.
} 
2019; emphasis added.) To this end, we contrasted two circumstances, both of which involved instructing people to entertain themselves with only their thoughts. Whereas one group (control group) was placed in an empty room, the other (affordances group) was placed in a room containing various objects that afforded meaningful engagement. Critically, participants in both conditions were asked to refrain from engaging with anything in either environment. For empty room this amounted to staying seated, awake and having only your thoughts. We hypothesized that participants placed in a room with avenues for meaningful engagement would report greater levels of boredom than those placed in an empty room, presumably because such environments dramatically increase the opportunity costs associated with the primary task. Furthermore, given that boredom has been characterized as a state of wanting and is thought to motivate the individual to engage in a more satisfying activity, we examined whether the exposure to different environments also affected people's state of wanting to engage. We hypothesized that environments with many affordances would elicit a greater desire for engagement (i.e., increased wanting) than environments with no affordances. Finally, since we prevented people from engaging in their environments, it seemed plausible that this manipulation would elevate feelings of frustration. However, we believed that our manipulation would primarily affect boredom-a state we believe is characterized by a failure to launch into goal pursuit (Mugon, Struk, \& Danckert, 2018). In contrast, frustration is a state that arises when we encounter obstacles during goal pursuit (Dollard, 1938). Thus, we measured feelings of frustration and predicted that there would be no differences in frustration between the engaging and non-engaging environments as neither circumstance impedes a goal in progress. 


\section{Method}

Pre-Registration. This study was preregistered on AsPredicted under the title of "The Role of Action Space on the Experience of Boredom under Constraints" (\#2577) on January 16, 2017 (http://aspredicted.org/blind.php?x=gh2jr7)

Participants. In a pilot study $(N=80)$, the primary effect size (the effect of condition on reported boredom) was Cohen's $d=0.42$. Thus, to attain $80 \%$ power, a sample of 90 participants per condition would be needed. Two-hundred and twenty-eight undergraduates (184 females, mean age $=20.35, S D=3.23)$ participated in exchange for partial course credit. In terms of selfreported ethnicity, $41.9 \%$ identified as White/Caucasian, $21.1 \%$ as East Asian, $14.5 \%$ as South Asian, 5.7\% as Middle Eastern, $4.8 \%$ as Southeast Asian, 3.1\% as Black/African, 3.1\% as West Indian/Caribbean, $2.6 \%$ as Hispanic, with $3.1 \%$ of the sample responding 'other' or declining to indicate the ethnicity they identified with. In accordance with our pre-registration, we preselected participants who were not highly prone to boredom in order to maximize power ${ }^{2}$. Short Boredom Proneness Scale (SBPS, Struk, Carriere, Cheyne \& Dankert, 2015) was used to measure boredom proneness, and was administered at the beginning of each academic term as part of a larger survey used for pre-screening purposes. Participants were qualified to participate in our study if their SBPS score was no more than a quartile above the group median. Participants were randomly assigned to one of two experimental conditions. Data were collected throughout the 2017 academic year and into the winter term of 2018. We decided, a priori, to collect data from

\footnotetext{
${ }^{2}$ In pilot work, a condition effect was observed including all participants regardless of scores on the SBPS. However, analyses indicated that the effect was greater $(\mathrm{d}=0.66)$ if highly boredom prone individuals (those whose SBPS scores were at least 34 or above $3^{\text {rd }}$ quartile) were removed. Thus, we made an a priori decision to exclude these participants as a way to increase power.
} 
as many participants as needed to attain 90 rule-complying participants per condition (see

Supplemental Material for compliance rules). This study received ethics clearance from University of Waterloo Office of Research Ethics (ORE\# 31108).

Procedure and experimental setup. Participants were either seated in (1) an empty room in which there was only a chair, an empty bookshelf, a chalk board with no chalk, a filing cabinet, and a desk (control condition) or (2) an engaging room in which, in addition to the items in the empty room, there were numerous objects with which participants could normally interact: chalk was added to the chalk board, a laptop computer in Firefox web-kiosk mode and a Google front page were opened, and a partially completed Lego car puzzle, a partly completed jig-saw puzzle, three sheets of blank paper, and a set of crayons were placed in the room (affordances condition). Participants in both conditions were instructed to refrain from engaging with their environment and to instead stay seated, remain awake, and entertain themselves with their thoughts for a 15-minute period. (To ensure that participants complied with this instruction, we placed a hidden camera — encased in a coffee mug; Lawmate Thermos Hidden DVR Spy Camera with 740p High Resolution - on top of a filing cabinet in the corner of the room.) Prior to the researcher's departure, participants were required to give their backpack and electronic devices to the researcher for the duration of the study (Wilson et al, 2014). After the 15-minute "just think" period, the researcher returned to administer a brief questionnaire, which was completed on a tablet. Participants responded to the following 3 questions: "Rate how bored you were over the last 15-minute period"; "Rate how frustrated you were over the last 15-minute period"; "Rate how much you wanted to do something over the last 15-minute period." Participants responded to all questions on a Likert scale ranging from, 1 ("Not At All”) to 9 ("Extremely"). Prior to departure, participants were informed of the purpose of the experiment, and the use of video 
recording was disclosed and consent to review the video recording was obtained. Following each participant's departure, the video recording was reviewed by the researcher and the participant was deemed a rule-breaker if they did any of the following: stood up, fell asleep, or interacted with their environment (these exclusion criteria can be found in our preregistration; see Supplemental Material for detailed coding instructions). The exclusion criteria were determined, a priori, based on participant feedback in a pilot study in which some participants occasionally broke rules when placed in a room with objects that they were told to refrain from engaging with. To compute the inter-rater reliability and to determine the final exclusions, following conclusion of data collection, two independent video coders reviewed the videos using the same coding instructions previously used by the experimenter staff during data collection (see Supplementary Material for detailed breakdown of coding results). Discrepancies between coders were resolved by having both coders meet and agree on a single coding outcome.

\section{Results}

Normality. All data analyses were conducted in R (R Core Team, 2015). Shapiro-Wilks tests of normality (Shapiro \& Wilk, 1965) indicated that none of the study variables were normally distributed (all $p$ s < 0.05). As such, non-parametric comparisons were conducted throughout. Independent-samples non-parametric comparisons were conducted using Wilcoxon's rank-sum test (Wilcoxon, 1945) ${ }^{3}$. Medians, first and third quartiles, means and standard deviations are reported to describe each variable. For all comparisons, p values (based on Mann-

\footnotetext{
${ }^{3}$ In our pre-registration, we indicated that we would conduct a two-sample permutation (exact significance) $t$ test if assumptions of normality were violated. However, we now realize that the Wilcox test is more appropriate. We report the results from both analyses here and note that results do not differ substantially and thus do not change any of our interpretations.
} 
Whitney-Wilcoxon test), non-parametric effect sizes (Cliff's delta) and parametric effect sizes (Cohen's D; assuming an independent samples t-test) are reported (Cliff, 1993; Cohen, 1988).

Condition Differences. Since a valid test of our hypothesis requires that participants follow our instructions, the following analyses were conducted on data from rule-followers only (Table 1 presents descriptive statistics for rule followers and for the full sample). A Bonferroni corrected family-wise alpha of $0.5 / 3=0.017$ was used for all comparisons. As predicted, participants reported greater levels of boredom in the affordances condition $(\mathrm{Med}=4)$ than in the empty room (control condition; $M e d=3 ; p=0.006$, Cliff's $D=0.23$, Cohen's $D=0.39$ ). There was no significant difference in wanting between the affordances condition $(M e d=5)$ and the empty room $(M e d=4 ; p=0.121$, Cliff's $D=0.13$, Cohen's $D=0.24)$. Likewise, there was no significant difference in frustration between the affordances condition $(\mathrm{Med}=2)$ and the empty room condition $(M e d=1 ; p=0.066$, Cliff's $D=0.15$, Cohen's $D=0.24)$.

$\boldsymbol{A N C O V A}$. To assess discriminatory validity of our manipulation's effect on boredom, we tested whether the effect of our manipulation remained after controlling for frustration. Following inclusion of frustration as a co-variate, our manipulation continued to have a significant effect on boredom, $F(1,179)=4.40, p=.037$. In line with this analysis, we also wanted to test whether our manipulation uniquely affects boredom beyond its effect on wanting. Thus, we repeated this analysis, this time controlling for wanting. Following inclusion of wanting as a co-variate, our manipulation continued to have a significant effect on boredom, $F(1$, $179)=4.80, p=.030$.

We found no significant effects of gender as a function of rule compliance (see Supplementary Material). Further exploratory analyses, including correlations between study variables and mediation analyses can be found in the Supplementary Material. 
[Table 1 about here]

\section{Discussion}

Consistent with the opportunity-cost model of boredom (see also Kurzban et al., 2013), when participants were asked to refrain from engaging with the environment and instead entertain themselves with their own thoughts, those placed in a room with many options for engagement (affordances condition) reported higher levels of boredom than those placed in an empty room. This suggests that having a dearth of options for engagement (i.e., being in an empty room, with only your thoughts for entertainment) is in fact less boring than being exposed to (but unable to interact with) numerous options for engagement. Presumably, this occurs because the task of entertaining oneself with one's own thoughts is associated with greater opportunity costs in an environment that is rich in potentially valuable or engaging alternatives.

Boredom is characterized as a state of wanting coupled with an inability to engage in a satisfying activity (Eastwood et al, 2012). Consistently we observed a strong relationship between boredom and wanting ( $r=0.77$; Supplementary Material). However, our manipulation did not have a significant effect on wanting alone. There are at least two possibilities for this null finding. First, the effect of our manipulation on wanting may have been substantially weaker than the effect on boredom making it more challenging to detect any influence on wanting with current design. Indeed, in the present study, we found preliminary evidence to suggest that exposure to alternative activities (i.e., increased opportunity costs) primarily affects boredom (beyond frustration or wanting), which precedes the desire to engage with the environment (see 
Supplementary Materials). This suggests that, any effect on wanting as a function of our manipulation may be secondary to the effect on boredom. Alternatively, we may fail to see a significant effect of our manipulation on wanting because boredom and wanting are independent; that is, an individual may become bored without experiencing an increase in wanting - an outcome that our manipulation may have inadvertently yielded. Under conditions of constraint, whether the effect of opportunity costs on wanting is weak, or whether boredom can be completely decoupled from wanting, is a line of inquiry worthy of investigation in future studies.

Since we regarded frustration as a state that arises when we encounter obstacles during goal pursuit -a condition that both of our rooms lacked since goals were explicitly not pursuedwe predicted that frustration would be unaffected by our manipulation. We found that participants in the affordances condition did not report significantly higher levels of frustration than those in the empty room. Unlike boredom, most people reported relatively little to no frustration in both conditions (i.e., all scores were on the low end of the scale; Table 1). Nevertheless, a further exploratory analysis indicated that wanting and boredom may contribute to feelings of frustration (see Supplementary Materials).

Our results dovetail well with functional theories of boredom that suggest that boredom signals dissatisfaction with the current situation and a desire to engage in a more satisfying activity (Elpidorou, 2018, 2018). These results are consistent with how we view trait-boredom proneness - a tendency to experience boredom more frequently and intensely (Farmer \& Sundberg, 1986). Boredom-prone individuals may see options for engagement, but fail to effectively launch into them (Mugon et al., 2018). We also speculate that the mechanism by which the state of boredom is elevated when options for engagement are present but restricted (as in our affordances condition), is via the representation of increased opportunity costs. This 
notion is consistent with prior research showing that high-boredom-prone individuals are more likely to be assessment oriented - placing an emphasis on evaluating and comparing alternatives for action (Higgins, Kruglanski, \& Pierro, 2003; Kruglanski et al., 2000; Struk, Scholer, \& Danckert, 2016; Mugon et al., 2018). Presumably, this kind of fruitless rumination over the potential options for engagement makes the opportunity costs associated with many (typically not boring) situations salient. In the current experiment we did not find that boredom proneness moderated the effect of our manipulation on the experience of state boredom (see Supplementary Materials). Since this may be due to the fact that we pre-selected participants who were not highly prone to boredom (to ensure sufficient power), future research should investigate whether boredom proneness acts as a moderator of state boredom as a function of the presence or absence of options for action (i.e., affordances).

On a practical level, our results suggest, somewhat counterintuitively, that being exposed to many outlets for engagement may have its downsides. That is, in the presence of opportunity costs (in this case, alternative activities) our current activity becomes boring, and hence, sustaining attention on the activity will likely prove difficult. This is particularly problematic when attainment of an adequate outcome is highly dependent on maintenance of engagement, and disengagement is not a viable option. For example, a student with a cellphone in his pocket may become more bored with a lecture relative to his peers who do not have access to a cellphone. This is because the opportunity costs associated with the lecture are more salient when one is equipped with a device that affords many alternative, satisfying activities. Despite 
the apparent opportunity cost, commitment to attending to the lecture material does not avail oneself of engaging in the satisfying alternative, and thus prevents the alleviation of boredom. ${ }^{4}$

Our findings have direct implications for reducing boredom in circumstances where opportunity-cost saliency is suspected as the primary cause. The solution for reducing boredom in such cases may be as simple as tailoring the environment in a way that limits access to, or eliminates the cues of, activities unrelated to the task of interest. In other words, the goal of such intervention should be to minimize the salience of opportunity costs associated with the primary task. This can be implemented as part of self-help program, for example deciding not to bring your cellphone to a lecture or choosing to study in a location that is different from that in which you recreate. Alternatively, interventions may be implemented on an organizational level, for example, by encouraging students to leave their cellphones at home or in their locker.

\section{Concluding Remarks}

In the present study, we found that the inability to engage with an environment that afforded many alternative activities promoted feelings of boredom and a desire to engage with the environment. These results corroborate the notion that activities characterized by high opportunity costs (i.e., presence of alternative activities with the potential to be more engaging than the primary task) contribute to the experience of boredom, which signals dissatisfaction with the current activity and a desire to engage in some alternative. Our findings suggest that in the presence of satisfying alternatives an activity may become boring, and hence, that boredom

\footnotetext{
${ }^{4}$ It is, however, important to note that access to alterative satisfying activities could help alleviate boredom in some contexts, such as those in which engagement is not necessary for task completion. The most common example of this would be waiting: waiting does not require sustained engagement and permits engagement in other satisfying alternatives, such as conversing with someone.
} 
could be alleviated by eliminating exposure to activities that are irrelevant to the primary task. Moving forward, we suggest that research should examine the effectiveness of interventions aimed at minimizing opportunity costs or its salience to combat boredom in ecologically valid contexts, such as workplace or classroom settings.

\section{Open Practices Statement}

The preregistration for this experiment can be accessed at https://aspredicted.org/p5b36.pdf . The materials, dataset generated and analyzed during the current study, as well as the analysis script, are available in the OSF repository https://osf.io/9j8uz/?view_only=3dc0221a888545c5ad93f15eb66b948a . 


\section{References}

Bench, S., \& Lench, H. (2013). On the function of boredom. Behavioral sciences, 3(3), 459-472. doi:10.3390/bs3030459

Cliff, N. (1993). Dominance statistics: Ordinal analyses to answer ordinal questions. Psychological bulletin, 114(3), 494. doi:10.1037/0033-2909.114.3.494

Cohen, J. (1988). Statistical power analysis for the behavioral sciences (2nd ed.). Hillsdale, NJ: Erlbaum. doi:10.4324/9780203771587

Custers, R. (2009). How Does Our Unconscious Know What We Want? In G. B. Moskowitz \& H. Grant (Eds.), The Psychology of Goals (pp. 180-181). New York, NY: The Guilford Press.

Danckert, J., Mugon, J., Struk, A., \& Eastwood, J. (2018). Boredom: What Is It Good For? In The Function of Emotions (pp. 93-119). Springer, Cham.

Dollard, J., Miller, N. E., Doob, L. W., Mowrer, O. H., \& Sears, R. R. (1939). Frustration and aggression. Yale University Press, 1939.

Eastwood, J. D., Frischen, A., Fenske, M. J., \& Smilek, D. (2012). The unengaged mind defining boredom in terms of attention. Perspectives on Psychological Science, 7(5), 482-495. doi:10.1177/1745691612456044

Elpidorou, A. (2018). The good of boredom. Philosophical Psychology, 31(3), 323-351. doi:10.1080/09515089.2017.1346240 
Elpidorou, A. (2018). The bored mind is a guiding mind: Toward a regulatory theory of boredom. Phenomenology and the Cognitive Sciences, 17(3), 455-484. doi:10.1007/s11097-017-9515-1

Farmer, R., \& Sundberg, N. D. (1986). Boredom proneness--the development and correlates of a new scale. Journal of Personality Assessment, 50(1), 4-17. doi:10.1207/s15327752jpa5001_2

Goetz, T., Frenzel, A. C., Hall, N. C., Nett, U. E., Pekrun, R., \& Lipnevich, A. A. (2014). Types of boredom: An experience sampling approach. Motivation and Emotion, 38(3), 401-419. doi: 10.1007/s11031-013-9385-y

Gomez-Ramirez, J., \& Costa, T. (2017). Boredom begets creativity: A solution to the exploitation-exploration trade-off in predictive coding. Biosystems, 162, 168-176. doi:10.1016/j.biosystems.2017.04.006

Higgins, E. T., Kruglanski, A. W., \& Pierro, A. (2003). Regulatory mode: Locomotion and assessment as distinct orientations. Advances in Experimental Social Psychology, 35, 293-344. doi:10.1016/S0065-2601(03)01005-0

Kruglanski, A. W., Higgins, E. T., Pierro, A., Thompson, E. P., Atash, M. N., Shah, J. Y., \& Spiegel, S. (2000). To "do the right thing" or to "just do it": Locomotion and assessment as distinct self-regulatory imperatives. Journal of Personality and Social Psychology, 79(5), 793-815. doi:10.1037/0022-3514.79.5.793

Kurzban, R., Duckworth, A., Kable, J. W., \& Myers, J. (2013). An opportunity cost model of subjective effort and task performance. Behavioral and Brain Sciences, 36(6), 661-679. doi:10.1017/S0140525X12003196 
Mugon, J., Struk, A., \& Danckert, J. (2018). A failure to launch: Exploring the self-regulatory profile of boredom. Frontiers in Psychology, 9, 1126. doi:10.3389/fpsyg.2018.01126

R Core Team. (2015). R: A language and environment for statistical computing [Computer software manual]. Vienna, Austria. Retrieved from http://www.R-project.org/

Shapiro, S. S., \& Wilk, M. B. (1965). An analysis of variance test for normality (complete samples). Biometrika, 52(3/4), 591-611. doi:10.2307/2333709

Struk, A. A., Carriere, J. S., Cheyne, J. A., \& Danckert, J. (2017). A short boredom proneness scale: Development and psychometric properties. Assessment, 24(3), 346-359. doi:10.1177/1073191115609996

Struk, A. A., Scholer, A. A., \& Danckert, J. (2016). A self-regulatory approach to understanding boredom proneness. Cognition and Emotion, 30(8), 1388-1401. doi:10.1080/02699931.2015.1064363

The National Center on Addiction and Substance Use (2003). Report on Teen Cigarette Smoking and Marijuana Use. Retrieved on Feb 4, 2019, from Center on Addiction Website: https://www.centeronaddiction.org/addiction-research/reports/report-teencigarette-smoking-and-marijuana-use

Westgate, E. C., \& Wilson, T. D. (2018). Boring thoughts and bored minds: The MAC model of boredom and cognitive engagement. Psychological Review, 125(5), 689-713. doi:10.1037/rev0000097

Wilcoxon, F. (1945). Individual comparisons by ranking methods. Biometrics bulletin, 1(6), 8083. doi: $10.2307 / 3001968$ 
Wilson, T. D., Reinhard, D. A., Westgate, E. C., Gilbert, D. T., Ellerbeck, N., Hahn, C., Brown C.L. \& Shaked, A. (2014). Just think: The challenges of the disengaged mind. Science, 345(6192), 75-77. doi:10.1126/science. 1250830

Affordance. (2019). In Oxford Online Dictionary. Retrieved from

https://en.oxforddictionaries.com/definition/affordance 
Table 1. Descriptive statistics and condition differences for rule-compliers and full sample. ${ }^{5}$

\begin{tabular}{|c|c|c|c|c|c|c|c|c|c|}
\hline & \multicolumn{3}{|c|}{$\begin{array}{l}\text { Control Condition } \\
n=91 \text { (108) }\end{array}$} & \multicolumn{3}{|c|}{$\begin{array}{c}\text { Affordances Condition } \\
n=91(121)\end{array}$} & \multirow[b]{2}{*}{$p$} & \multirow[b]{2}{*}{$\begin{array}{c}\text { Cliff's } \\
D\end{array}$} & \multirow[b]{2}{*}{$\begin{array}{c}\text { Cohen's } \\
D\end{array}$} \\
\hline & $Q 1|Q 2| Q 3$ & $M$ & $S D$ & $Q 1|Q 2| Q 3$ & $M$ & $S D$ & & & \\
\hline \multirow{2}{*}{ Boredom } & $2|3| 5$ & 3.62 & 2.16 & $3|4| 6$ & 4.47 & 2.20 & .006 & 0.23 & 0.39 \\
\hline & $2|3| 5.25$ & 3.66 & 2.20 & $3|4| 6$ & 4.46 & 2.31 & .006 & 0.21 & 0.35 \\
\hline \multirow{2}{*}{ Wanting } & $2|4| 6$ & 4.43 & 2.34 & $3|5| 7.5$ & 5.01 & 2.53 & .121 & 0.13 & 0.24 \\
\hline & $2|4| 7$ & 4.56 & 2.34 & $3|5| 7$ & 5.11 & 2.60 & .119 & 0.12 & 0.22 \\
\hline \multirow{2}{*}{ Frustration } & $1|1| 2$ & 1.79 & 1.20 & $1|2| 3$ & 2.12 & 1.53 & .066 & 0.15 & 0.24 \\
\hline & $\mathbf{1}|\mathbf{1}| \mathbf{2}$ & 1.80 & 1.25 & $1|2| 3$ & 2.31 & 1.71 & .005 & 0.22 & 0.34 \\
\hline
\end{tabular}

Note. Statistics for the full sample are presented in bold; statistics for rule-followers are nonbolded. $Q 2=$ median, $Q 1=$ first quartile, $Q 3=$ third quartile, $\mathrm{M}=$ mean, $\mathrm{SD}=$ standard deviation, $p=$ significance value of Wilcoxon's rank-sum test

\footnotetext{
${ }^{5}$ In our pre-registration, we indicated that a two-sample permutation (exact significance) $t$ test would be conducted. Although we instead opted to use the Wilcoxon Ranked-Sum test, we report significance values from the originally planned analyses here: $p=.010$ (.011) for boredom; $p=.014$ (.085) for wanting; $p=.121$ (.101) for frustration. We also report results from a regular t-test: $p=.009(.008)$ for boredom; $p=.109(.096)$ for wanting; $p=.106(.010)$ for frustration.
} 


\section{Supplementary Material}

Video Coding. Coding criteria used for video review and frequency of each criterion used is described in Table 1S. Inter-rater reliability between the two coders was (92.6\%).

Table 1S. Coding criterion and frequency of used criterion in each condition.

\begin{tabular}{|c|c|c|c|}
\hline Decision & Description & $\begin{array}{l}\text { Control } \\
\text { Condition } \\
\text { Frequency }\end{array}$ & $\begin{array}{l}\text { Affordances } \\
\text { Condition } \\
\text { Frequency }\end{array}$ \\
\hline Complied & $\begin{array}{l}\text { Participant followed instructions and entertained } \\
\text { themselves with their own thoughts, they did not engage } \\
\text { with the environment, they did not stand up from their seat, } \\
\text { nor did they fall asleep. Use this option if participants did } \\
\text { not fit any of the following criteria }\end{array}$ & 91 & 91 \\
\hline Stand & Participant stood up from the chair & 7 & 2 \\
\hline Sleep & $\begin{array}{l}\text { Participant fell asleep. Not only does participant need to } \\
\text { close their eyes, their body posture needs to become visibly } \\
\text { slumped; shoulders and head lean down. If participant rests } \\
\text { head on table, regard this as sleeping. Exempt closed eyes } \\
\text { only. }\end{array}$ & 1 & 4 \\
\hline Engage & $\begin{array}{l}\text { Participant interacted with the environment. (i.e., touched } \\
\text { any of the objects in the room: Laptop, Jigsaw puzzle, } \\
\text { Lego Car puzzle, Paper and Crayons.) }\end{array}$ & N/A & 15 \\
\hline Deviate & $\begin{array}{l}\text { Participant remained seated but deviated from original } \\
\text { sitting position (no longer in front of the table and/or no } \\
\text { longer visible on camera). }\end{array}$ & 7 & 5 \\
\hline Missing & Video missing or partial. & 1 & 4 \\
\hline
\end{tabular}




\section{Results}

Gender differences. The following differences were analyzed on rule compliers only. Gender proportions did not significantly differ across conditions $\chi^{2}(1)<.001, p=1.00$. A Bonferroni corrected family-wise alpha of $0.5 / 3=0.017$ was used for all comparisons. There were no differences in reported boredom between females $(\mathrm{Med}=4)$ and males $(\mathrm{Med}=3 ; p=$ .149 , Cliff's $D=0.15$, Cohen's $D=0.30$ ). There were no differences in reported wanting levels between females $(\mathrm{Med}=5)$ and males $(\mathrm{Med}=4 ; p=.055$, Cliff's $D=0.20$, Cohen's $D=0.37)$. There were no differences in reported frustration levels between females $($ Med $=1)$ and males $($ Med $=1 ; p=.377$, Cliff's $D=0.09$, Cohen's $D=0.26)$.

Rule Breakers. Out of the 107 participants in the empty room, $16(15 \%)$ broke the rules, whereas, out of the 121 participants in the engaging room, $30(25 \%)$ broke the rules. First, we tested whether there were more rule breakers in the engaging room than the empty room. We found that this was not the case $\left(\chi^{2}(1)=2.84, p=0.092\right)$. Given that we could not randomly assign participants to break rules, the number of rule breakers versus non-rule breakers is highly uneven, and none of our variables are normally distributed, we could not conduct an appropriate factorial analysis. Instead, we conducted a number of point-biserial correlations between rule breaking and our study variables, for each condition, (Family-wise alpha $=0.05 / 6=.008$ ). Neither in empty room nor in the affordances condition was there any difference in any of the study variables between those who broke rules or complied $(\mathrm{p}>.031)$.

Correlational analyses. Our analysis indicated that boredom was strongly correlated with wanting and frustration. Likewise, frustration was strongly correlated with wanting (Table S2). Using DeCoster's (2005) method of comparing two dependent correlations, we observed that the correlation between boredom and wanting was significantly stronger than the correlation 
between boredom and frustration, $Z=4.91, p<.001$. Boredom proneness was not correlated with any of the study variables.

Table S2. Correlation matrix for all study variables.

\begin{tabular}{llll}
\hline & Frustration & Wanting & Boredom Proneness \\
\hline Boredom & $\mathbf{0 . 5 3 ( 0 . 5 5 )}$ & $\mathbf{0 . 7 7}(\mathbf{0 . 7 7})$ & $0.06(0.02)$ \\
Frustration & & $\mathbf{0 . 5 1}(\mathbf{0 . 5 4})$ & $-0.06(-0.09)$ \\
Wanting & & $0.07(0.09)$ \\
\hline
\end{tabular}

Correlations of compliers only (full sample). All bolded correlations are significant at $p<.001$

Boredom Proneness. We tested whether addition of boredom proneness as a covariate and a moderator affected the relationship between our manipulation and all study variables. Boredom proneness did not significantly moderate the effect of condition on state reports of boredom $(p=0.580)$, furthermore condition remained a significant predictor of boredom following inclusion of boredom proneness and the boredom proneness by condition interaction term as covariates $(p=.010)$. Boredom proneness did not significantly moderate the effect of condition on frustration $(p=.918)$, condition continued to be a non-significant predictor of frustration following inclusion of boredom proneness and the boredom proneness by condition interaction term as covariates $(p=.100)$. Boredom proneness did not significantly moderate the effect of condition on wanting ( $p=.864)$, condition continued to be a non-significant predictor of wanting following inclusion of condition and the boredom proneness by condition interaction term as covariates $(p=.123)$.

Mediation analyses. As part of our exploratory analyses, we tested the possibility that the effect of our manipulation on wanting was mediated by boredom. Furthermore, given that 
frustration was correlated with boredom and wanting we opted to conduct additional exploratory analyses investigating whether wanting mediated the effect of boredom on frustration. The following analyses were performed on the rule-following subsample only. To investigate all study variables simultaneously a structural equation model was constructed as depicted in Figure S1 with the aid of lavaan R package (Rosseel, 2012). Here it is assumed that frustration is an outcome of failure to resolve boredom and failure to act on desires (wanting), thus frustration appears as the final variable within the model. Direct and indirect effects were estimated using bootstrapping (with bias-corrected and accelerated (BCa) interval estimation). 10,000 Monte Carlo simulations were used to estimate parameters. Standardized direct effects are reported in Figure $\mathrm{S} 1$. The effect of condition on wanting was mediated by boredom $(\beta=0.15, p=.008)$ and condition had no residual direct effect on wanting $(\beta=0.03, p=.505)$. The effect of boredom on frustration was mediated by wanting $(\beta=0.20, p=.004)$, however boredom itself continued to have a significant effect on frustration $(\beta=0.32, p=.004)$. The indirect path between condition and frustration involving both, boredom and wanting, was marginally significant $(\beta=0.04, p=$ $.059)$.

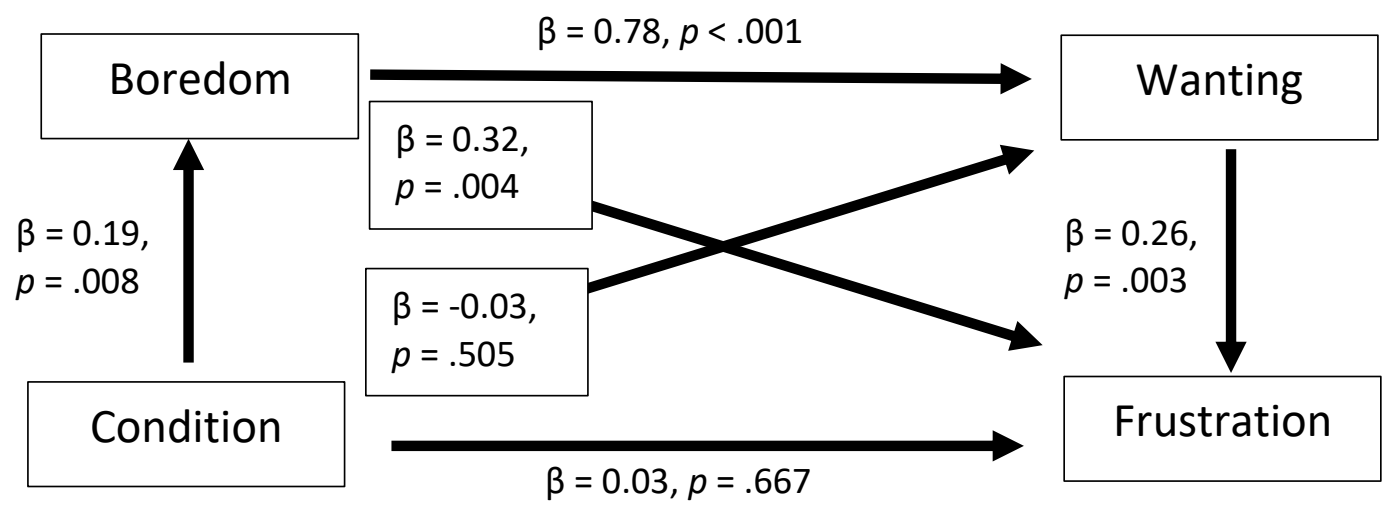




\section{References}

DeCoster (2005) Applied Linear Regression. Retrieved from http://www.stathelp.com/notes.html (pp. 13-14)

Rosseel Y (2012). "lavaan: An R Package for Structural Equation Modeling." Journal of Statistical Software, 48(2), 1-36. http://www.jstatsoft.org/v48/i02/. 